\title{
Incidence, risk factors, and outcome of Clostridioides difficile infection following urological surgeries
}

\author{
Kevin A. Nguyen ${ }^{1,2} \cdot$ Danny Q. Le ${ }^{1} \cdot$ Yvonne T. Bui $^{2}$. Sonali D. Advani ${ }^{3}$. Joseph Renzulli II ${ }^{4}$ Patrick A. Kenney ${ }^{4}$. \\ Michael S. Leapman ${ }^{4,5}$
}

Received: 25 July 2020 / Accepted: 5 December 2020 / Published online: 20 January 2021

(c) The Author(s), under exclusive licence to Springer-Verlag GmbH, DE part of Springer Nature 2021

\begin{abstract}
Purpose To assess the incidence, risk factors, and clinical outcomes associated with (Clostridioides difficile infection) CDI following urological surgery, which is the leading cause of nosocomial diarrhea and a growing public health burden.

Methods We queried the National Surgical Quality Improvement Program (NSQIP) to identify patients undergoing urological surgery in 2015-2016. We evaluated the 30-day incidence and factors associated with postoperative CDI and 30-day hospital readmission and length of stay as secondary outcomes. Among the subset of patients undergoing radical cystectomy with urinary diversion (surgery with highest CDI incidence) we used multivariable logistic regression analysis to evaluate independent clinical and demographic factors associated with postoperative CDI.

Results We identified 98,463 patients during the study period. The overall 30-day incidence of CDI was $0.31 \%$, but varied considerably across surgery type. The risk of CDI was greatest following radical cystectomy with urinary diversion $(2.72 \%)$ compared to all other urologic procedures $(0.19 \%)$ and was associated with increased risk of hospital readmission $(p<0.0001)$, re-operation $(p<0.0001)$, and longer mean length of stay $(p<0.0001)$ in this cohort. Among patients undergoing radical cystectomy with urinary diversion, multivariable logistic regression revealed that preoperative renal failure (OR: 5.30, 95\% CI 1.13-24.9, $p=0.035$ ) and blood loss requiring transfusion (OR: 1.67, 95\% CI 1.15-2.44, $p=0.0075$ ) were independently associated with CDI.

Conclusions In a nationally representative cohort, the incidence of CDI was low but varied substantially across surgery types. CDI was most common following radical cystectomy and associated with potentially modifiable factors such as blood transfusion and significantly longer length of stay.
\end{abstract}

Keywords Clostridioides difficile $\cdot$ Radical cystectomy $\cdot$ Infection $\cdot$ Surgical outcomes $\cdot$ Length of stay

Supplementary Information The online version contains supplementary material available at https://doi.org/10.1007/s0034 5-020-03551-y.

Michael S. Leapman

michael.leapman@yale.edu

1 David Geffen School of Medicine, University of California Los Angeles, Berkeley, USA

2 Mayo Clinic Alix School of Medicine, Rochester, USA

3 Department of Medicine, Duke University School of Medicine, Durham, USA

4 Department of Urology, Yale University School of Medicine, New Haven, USA

5 Yale Cancer Outcomes Public Policy and Effectiveness Research (COPPER) Center, New Haven, USA

\section{Abbreviations}

CDI Clostridioides difficile Infection

NSQIP National Surgical Quality Improvement Program

ICU Intensive care unit

CPT Common procedural terminology

ASA American Society of Anesthesiology

\section{Introduction}

Clostridioides difficile (C. difficile), formerly known as Clostridium difficile, is a spore-forming, gram-positive anaerobic bacillus that colonizes the human gut and is one of the leading causes of opportunistic nosocomial infection in the United States $[1,2]$. C. difficile infection (CDI) can cause a wide spectrum of clinical features ranging from asymptomatic colonization to diarrhea, hemorrhage, 
pseudomembranous colitis, and death [3, 4]. Although there have been significant advancements in infection prevention, the incidence of CDI has substantially increased since the early 2000s with the emergence of a hypervirulent strain of $C$. difficile ribotype 027 that releases much higher toxin levels earlier in the growth phase than other variants [2].

There has been increasing awareness of the impact of CDI on perioperative outcomes. Several risk factors for CDI identified in the general surgery literature include use of broad-spectrum antibiotics, advanced age, and immunosuppression [5]. In addition, prior publications have indicated that CDI is associated with decreased patient reported quality of life measures, increased hospital length of stay, intensive care unit (ICU) admissions, postoperative emergency room utilization, and readmissions [4, 6, 7]. In cost analyses, CDI contributed an additional $\$ 21,000$ in costs per patient, resulting in an estimated national burden of $\$ 7$ billion per year [8]. Given direct clinical and economic burdens of CDI, it is increasingly important to understand and address the issues surrounding CDI prevention.

Nosocomial infections are significant contributors to adverse patient outcome and increased healthcare expenditure after urological surgery [9]. Yet, little information exists in the literature regarding the effects of CDI after urological surgery. Previous retrospective, single-institution studies have begun to characterize the risk of CDI after urologic surgeries of larger scale, such as radical cystectomy [9]. However, to our knowledge, no cross-sectional national analyses have been performed to study this question across the span of common urological surgeries. Therefore, we aimed to comprehensively evaluate the incidence, risk factors, and postoperative complications associated with CDI following urological surgery using the American College of Surgeons National Surgical Quality Improvement Project (NSQIP).

\section{Methods}

\section{Study population}

The American College of Surgeons National Surgical Quality Improvement Program (NSQIP) was queried to identify records from 2015 through 2016. NSQIP consolidates clinical data from patients undergoing surgical procedures in the United States at more than 200 hospitals. The data collected encompass more than 200 variables, which include pre-operative, perioperative/intraoperative, postoperative complications, demographic, comorbidity, and mortality outcomes 30 days after surgery. Surgical staff reviewers at each hospital site curate the data using a standardized protocol and regularly audit participating sites. Patients were diagnosed with any of the following diagnostic laboratory tests: stool culture, glutamate dehydrogenase enzyme immunoassay or latex agglutination, PCR assay, stool cytotoxin test, or enzyme immunoassay for toxins A and B. All patients diagnosed with CDI were treated by either oral and/or IV antibiotics, fecal enemas, or surgical management for fulminant/ uncontrolled C. diff. colitis.

We included patients who underwent urological surgery between January 1, 2015 and December 31, 2016, based on the availability of CDI coding within NSQIP. We used common procedural terminology (CPT) codes to classify major procedures (Supplementary Table 1). The primary outcome of interest was CDI infection as documented within 30-days of the procedure.

Clinicopathologic and demographic variables including age, sex, operative time, total length of stay duration, race, comorbidities (history of bleeding disorder, ascites, congestive heart failure, chronic obstructive pulmonary disease, diabetes, hypertension, smoking, steroid/immunosuppressant usage), preoperative conditions (renal failure, dialysis, transfusion), sex, American Society of Anesthesiology (ASA) Class, disseminated cancer status, intraoperative bleeding requiring transfusion, mortality, 30-day readmission, and 30-day reoperation records were evaluated. A preplanned subgroup analysis was then performed to assess differences in clinicopathologic, demographic, and outcome measurements in patients who received radical cystectomy due to the high incidence of CDI in this population documented in the literature [10].

\section{Statistical analysis}

We used the chi-square test and Student t-tests to evaluate differences in demographic and clinicopathologic characteristics between patients who developed CDI and those who did not. The Anderson-Darling test was used to verify normality of distribution. Given the substantially higher incidence of CDI in patients undergoing radical cystectomy, we constructed multivariable logistic regression models in forward-step fashion to evaluate independent predictors of developing postoperative CDI in this patient subset. We applied a Bonferroni correction to account for multiple comparisons. Data analysis was performed using JMP Pro v.11.1 statistical software (SAS Institute Inc., Cary, NC, USA) and $\mathrm{R}$ version 3.5.1.

\section{Results}

\section{CDI following urological surgery}

Of 98,463 patients undergoing urological surgery within NSQIP, we identified $306(0.31 \%)$ cases of postoperative CDI (Table 1). CDI was more common after radical cystectomy with urinary diversion $(2.72 \%)$ compared to all 
Table 1 Comparison of Demographics and Clinicopathologic Characteristics between Patients with and without C. difficile infection following urological surgeries

\begin{tabular}{|c|c|c|c|}
\hline & $\begin{array}{l}\text { C. difficile } \\
306\end{array}$ & $\begin{array}{l}\text { No } C \text {. difficile } \\
98,157\end{array}$ & $p$ value \\
\hline Procedure & & & $<0.0001$ \\
\hline Radical cystectomy & $123(2.72 \%)$ & $4397(97.28 \%)$ & \\
\hline All other urological procedures & $183(0.19 \%)$ & $93,760(99.81 \%)$ & \\
\hline ASA class & & & $<0.0001$ \\
\hline ASA classes 1-2 & $64(20.92 \%)$ & $47,105(47.99 \%)$ & \\
\hline ASA classes 3-5 & $242(79.08 \%)$ & $50,759(51.71 \%)$ & \\
\hline None assigned & $0(0 \%)$ & $293(0.3 \%)$ & \\
\hline Race & & & 0.2781 \\
\hline American Indian or Alaska native & $1(0.33 \%)$ & $387(0.39 \%)$ & \\
\hline Asian & $4(1.31 \%)$ & $2516(2.56 \%)$ & \\
\hline Black or African American & $19(6.21 \%)$ & $7957(8.11 \%)$ & \\
\hline Native Hawaiian or Pacific Islander & $3(0.98 \%)$ & $371(0.38 \%)$ & \\
\hline Unknown/not reported & $59(19.28 \%)$ & $19,431(19.80 \%)$ & \\
\hline White & $220(71.90 \%)$ & $67,495(68.76 \%)$ & \\
\hline Disseminated cancer & & & $<0.0001$ \\
\hline No & $275(89.87 \%)$ & $95,510(97.30 \%)$ & \\
\hline Yes & $31(10.13 \%)$ & $2647(2.70 \%)$ & \\
\hline Functional health status prior to surgery & & & 0.0092 \\
\hline Independent & $293(95.75 \%)$ & $95,770(97.57 \%)$ & \\
\hline Partially dependent & $11(3.59 \%)$ & $1451(1.48 \%)$ & \\
\hline Totally dependent & $2(0.65 \%)$ & $301(0.31 \%)$ & \\
\hline Unknown & $0(0 \%)$ & $635(0.65 \%)$ & \\
\hline History of ascites & & & 0.0115 \\
\hline No & $304(99.35 \%)$ & $98,106(99.95 \%)$ & \\
\hline Yes & $2(0.65 \%)$ & $51(0.05 \%)$ & \\
\hline History of bleeding disorders & & & 0.0007 \\
\hline No & $287(93.79 \%)$ & $95,627(97.42 \%)$ & \\
\hline Yes & $19(6.21 \%)$ & $2530(2.58 \%)$ & \\
\hline History of CHF & & & 0.0922 \\
\hline No & $301(98.37 \%)$ & $97,477(99.31 \%)$ & \\
\hline Yes & $5(1.63 \%)$ & $680(0.69 \%)$ & \\
\hline History of COPD & & & 0.157 \\
\hline No & $284(92.81 \%)$ & $92,972(94.72 \%)$ & \\
\hline Yes & $22(7.19 \%)$ & $5185(5.28 \%)$ & \\
\hline History of diabetes & & & 0.1066 \\
\hline No & $260(84.97 \%)$ & $79,970(81.47 \%)$ & \\
\hline Yes & $46(15.03 \%)$ & $18,187(18.53 \%)$ & \\
\hline History of hypertension & & & 0.0363 \\
\hline No & $118(38.56 \%)$ & $43,665(44.48 \%)$ & \\
\hline Yes & $188(61.44 \%)$ & $54,492(55.52 \%)$ & \\
\hline History of smoking & & & 0.389 \\
\hline No & $250(81.7 \%)$ & $82,013(83.55 \%)$ & \\
\hline Yes & $56(18.3 \%)$ & $16,144(16.45 \%)$ & \\
\hline History of steroid/immunosuppressant use for chronic condition & & & 0.0343 \\
\hline No & $291(95.1 \%)$ & $95,493(97.29 \%)$ & \\
\hline Yes & $15(4.90 \%)$ & $2664(2.71 \%)$ & \\
\hline
\end{tabular}


Table 1 (continued)

C. difficile

306

Intraoperative/postoperative bleeding requiring transfusion

No complication

Transfusions/intraop/postop

Preoperative dialysis

No

Yes

Preoperative renal failure

No

Yes

Preoperative systemic sepsis

None

Sepsis

Septic shock

Sirs

Preoperative transfusion

No

Yes

Sex

Female

Male

Ventilator dependence

No

Yes
$215(70.26 \%)$

$91(29.74 \%)$

$298(97.39 \%)$

$8(2.61 \%)$

$300(98.04 \%)$

$6(1.96 \%)$

$294(96.08 \%)$

$3(0.98 \%)$

$1(0.33 \%)$

$8(2.61 \%)$

$301(98.37 \%)$

$5(1.63 \%)$

$85(27.78 \%)$

$221(72.22 \%)$

$304(99.35 \%)$

$2(0.65 \%)$
No C. difficile

98,157

$p$ value

$<0.0001$

$93,500(95.26 \%)$

4657 (4.74\%)

0.0181

$97,179(99.00 \%)$

$978(1.00 \%)$

$97,828(99.66 \%)$

$329(0.34 \%)$

97,231 (99.06\%)

$188(0.19 \%)$

$38(0.04 \%)$

$700(0.71 \%)$

0.0832

$97,496(99.33 \%)$

$661(0.67 \%)$

$<0.0001$

$18,106(18.45 \%)$

$80,051(81.55 \%)$

0.007 other urological procedures $(0.19 \%)$ (Fig. 1). Univariate analysis revealed numerous factors associated with postoperative CDI including higher ASA class, presence of disseminated cancer, dependent health status (partially or totally) prior to surgery, history of ascites, history of bleeding disorders, hypertension, steroid/immunosuppressant use for chronic conditions, intraoperative/postoperative bleeding requiring transfusion, preoperative dialysis, preoperative renal failure, preoperative systemic sepsis, female sex, and ventilator dependence. For patients who received a radical cystectomy, we did not observe a statistically significant difference in CDI incidence for those who had either malignant or benign disease etiology $(2.8 \%$ vs. $1.89 \%, p=0.28$ ). For patients diagnosed with postoperative CDI, mortality was $6.86 \%$ compared to $0.62 \%$ among those without CDI.

Multivariable logistic regression revealed that the presence of disseminated cancer (OR: 2.05, 95\% CI 1.38-3.04, $p=0.0003)$, radical cystectomy with urinary diversion (OR: 8.02, 95\% CI 6.06-10.6, $p<0.0001)$, higher ASA class status (OR: 2.22, 95\% CI 1.64-3.01, p <0.0001, and intraoperative/postoperative bleeding (OR: $2.16,95 \%$ CI 1.59-2.94, $p<0.0001)$ were independently associated with postoperative CDI (Table 2).

\section{Incidence of CDI after radical cystectomy and urinary diversion}

Among patients who underwent radical cystectomy with urinary diversion, those with postoperative CDI had longer mean lengths of stay (14.6 vs. 9.4 days, $p<0.0001)$, however there were no differences in 30-day readmission $(24.4 \%$ vs. $20.5 \%, p=0.302)$ or re-operation $(7.3 \%$ vs. $5.4 \%, p=0.382)$. On univariate analysis, CDI after radical cystectomy was associated with intraoperative/ postoperative bleeding requiring transfusion $(52.0 \%$ vs. $36.2 \%, p<0.0001)$ and female sex $(33.3 \%$ vs. $24.2 \%$, $p=0.041$ ) (Supplementary Table 2). On multivariable logistic regression analysis evaluating for determinants of $\mathrm{CDI}$ in patients receiving radical cystectomy, preoperative renal failure (OR: 5.30, 95\% CI 1.13-24.9, $p=0.035$ ), intraoperative/postoperative bleeding requiring transfusion (OR: $1.67,95 \%$ CI 1.15-2.44, $p=0.0075$ ), and ventilator dependence (OR: 17.9 (1.69-189, $p=0.017$ ) were associated with the development of postoperative CDI (Supplementary Table 3). 


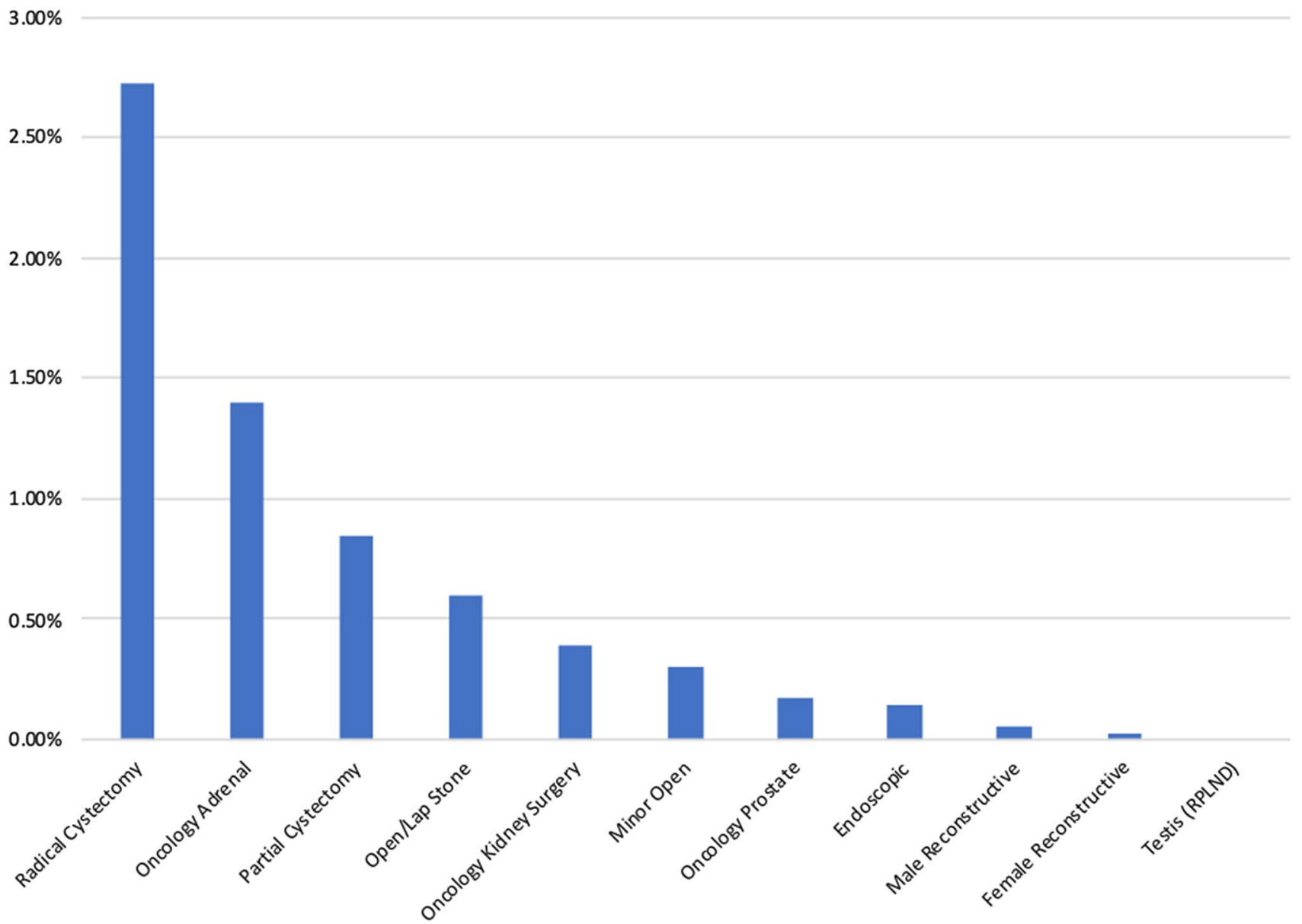

Fig. 1 Comparison of CDI rates by urological surgery procedures

\section{Discussion}

Using national hospital data, we evaluated the incidence, risk factors, and complications associated with CDI following urological surgery. Compared to previous literature, we found that the overall incidence of CDI was relatively low across all types of urological surgery; however, the incidence was more than ten-fold greater following radical cystectomy with urinary diversion. Moreover, cystectomy was associated with substantially higher incidence relative to other major oncologic surgeries. Controlling for surgery type, CDI was associated with both patient related factors, including female sex, and history of renal failure, ascites, bleeding disorders, hypertension, steroid/ immunosuppressant use, and systemic sepsis and treatment related factors such as longer operative time and receipt of blood transfusion. CDI after radical cystectomy with urinary diversion was associated with longer length of stay but not risk for readmission. By offering the largest crosssectional evaluation of CDI after urological surgery, this work provides additional information and external validity regarding the scope and potentially modifiable factors associated with this outcome.

$\mathrm{CDI}$ is well known to be associated with antibiotics use as well as other host factors that increase risk in the postoperative period [11]. The 'normal' human colonic flora offers protection against $C$. difficile over growth and, thereby, opportunistic infection. Use of antibiotics, especially betalactams or clindamycin, disrupts the typical state of the colonic microbiome and creates a susceptible environment for CDI, especially in the presence of risk factors [12]. Our data also reveal that specific clinical conditions were associated with higher risk of postoperative CDI including higher ASA class status and higher intraoperative/postoperative blood loss. Among the subset of patients undergoing radical cystectomy, risk factors associated with CDI included prior renal failure, ventilation dependence, and blood transfusions, a finding which has not yet been demonstrated in the urological literature [1, 13, 14]. Evidence from mouse models indicate that antibiotic treatment disrupts the microbiome of stable murine carriers, leading to development of a spore shedding state and increased transmission to other mice [15]. 
Table 2 Multivariate Logistic Regression model examining factors associated with postoperative CDI

\begin{tabular}{|c|c|c|}
\hline Variable & OR $(95 \% \mathrm{CI})$ & $p$ value \\
\hline Age & $1.02(1.01-1.03)$ & 0.0417 \\
\hline ASA Class & & $<0.0001$ \\
\hline ASA Class $1 / 2$ & 1.00 (Ref) & \\
\hline ASA Class $3-5$ & $2.22(1.64-3.01)$ & \\
\hline Major procedure & & $<0.0001$ \\
\hline All other urological procedures & $1.00(\operatorname{Ref})$ & \\
\hline Radical cystectomy with urinary diversion & $8.03(6.06-10.6)$ & \\
\hline Disseminated cancer & & 0.0003 \\
\hline No & $1.00(\operatorname{Ref})$ & \\
\hline Yes & $2.05(1.39-3.04)$ & \\
\hline \multicolumn{3}{|l|}{ Functional health prior to surgery } \\
\hline Independent & $1.00(\operatorname{Ref})$ & \\
\hline Partially dependent & $1.35(0.685-2.66)$ & 0.386 \\
\hline Totally dependent & $0.822(0.193-3.50)$ & 0.791 \\
\hline History of ascites & & 0.0681 \\
\hline No & 1.00 (Ref) & \\
\hline Yes & $4.21(0.90-19.7)$ & \\
\hline History of bleeding disorders & & 0.0191 \\
\hline No & $1.00(\operatorname{Ref})$ & \\
\hline Yes & $1.79(1.10-2.91)$ & \\
\hline History of CHF & & 0.227 \\
\hline No & 1.00 (Ref) & \\
\hline Yes & $1.75(0.705-4.37)$ & \\
\hline History of COPD & & 0.667 \\
\hline No & $1.00(\operatorname{Ref})$ & \\
\hline Yes & $0.904(0.57-1.43)$ & \\
\hline History of diabetes & & 0.0064 \\
\hline No & 1.00 (Ref) & \\
\hline Yes & $0.635(0.459-0.88)$ & \\
\hline History of hypertension & & 0.669 \\
\hline No & 1.00 (Ref) & \\
\hline Yes & $0.946(0.736-1.22)$ & \\
\hline History of smoking & & 0.941 \\
\hline No & 1.00 (Ref) & \\
\hline Yes & $0.988(0.729-1.34)$ & \\
\hline History of chronic steroid/immunosuppressant usage & & 0.370 \\
\hline No & $1.00(\operatorname{Ref})$ & \\
\hline Yes & $1.28(0.749-2.18)$ & \\
\hline Bleeding requiring transfusion & & $<0.0001$ \\
\hline No complication & $1.00(\operatorname{Ref})$ & \\
\hline Transfusions/Intraop/Post-op & $2.16(1.59-2.94)$ & \\
\hline Preoperative renal failure & & 0.0093 \\
\hline No & 1.00 (Ref) & \\
\hline Yes & $3.28(1.34-8.04)$ & \\
\hline Preoperative dialysis & & 0.3313 \\
\hline No & 1.00 (Ref) & \\
\hline Yes & $1.45(0.683-3.09)$ & \\
\hline Ventilator dependence & & 0.0146 \\
\hline No & $1.00(\operatorname{Ref})$ & \\
\hline Yes & $6.79(1.46-31.6)$ & \\
\hline Sex & & 0.0148 \\
\hline
\end{tabular}


Table 2 (continued)

\begin{tabular}{lll}
\hline Variable & OR $(95 \% \mathrm{CI})$ & $p$ value \\
\hline Male & $1.00($ Ref $)$ & \\
Female & $1.38(1.07-1.80)$ & \\
\hline
\end{tabular}

Stopping antibiotic treatment in mice led to suppression of C. difficile levels and recovery of the microbiome, which supports early cessation of prophylactic antibiotic treatment for all patients particularly those at highest risk. This data also raise a greater emphasis on modifiable provider interventions, such as prompt diagnosis with sensitive screening and subsequent confirmatory tests, handwashing with soap and water instead of alcohol-based agents, and contact precautions for at risk individuals.

Other proposed theories include direct effects on gut flora and antitoxin production that protect hosts against CDI [16]. As renal failure and ventilator dependence are indicative of serious systemic illness, it is plausible that altered immunological responses could be contributors [17, 18]. Moreover, blood transfusion may also suppress immunological responses increasing the risk of CDI [19]. Perioperative transfusion has been associated with an increased risk of surgical site infections (SSI), largely due to transfusionrelated immunomodulation. Perioperative blood transfusion may increase risk of postoperative CDI in a similar manner by modulating and suppressing the host immune system, especially circulating lymphocytes, as well as increased antibiotic exposure from higher incidence of SSI [20]. Thus, efforts such as preoperative control of anemia; the use of judicious transfusion thresholds; the minimization of intraoperative blood loss and the use of autologous blood products should be made to implement measures to avoid unnecessary transfusions [21].

These findings also suggest that careful considerations be noted toward interventions to where the risk is greatest, in particular radical cystectomy with urinary diversion [22]. The current AUA and EUA guidelines recommend perioperative antibiotic prophylaxis in cystectomy, which may encompass a second or third generation cephalosporin in addition to metronidazole [23, 24]. Given the significant risk of developing postoperative CDI with the use of prolonged cephalosporins [25], our findings imply the need for greater scrutiny when assessing the length of antibiotic use. While the AUA and EUA guidelines recommend $24 \mathrm{~h}$ of antibiotic coverage, the American College of Surgeons guidelines recommend stopping perioperative antibiotics after skin closure [26]. This recommendation can be applied in high risk individuals to reduce the risk of developing postoperative CDI. In radical cystectomy, the higher incidence of CDI may be related to the complex nature of the operation, bowel preparation, prolonged hospitalization, use of perioperative broader spectrum antibiotics, and intestinal diversion. Approximately $76 \%$ of postoperative CDI in the United States occur after hospital discharge, and the incidence of CDI may be linked to the observation that slower growth rates correlate with increased disease severity [27, 28]. Initiatives to reduce CDI rates with judicious antibiotic use are warranted, and we identified potentially modifiable factors that were associated with CDI after radical cystectomy including length of operation and blood loss/transfusion. Although we cannot imply a simple causal relationship, these factors have also been identified in other studies as associated with surgical site infections and appear to warrant additional investigation [29].

We found that CDI is independently associated with other adverse perioperative outcomes, including length of stay and readmission, which is consistent with previous literature [30]. Financial analysis of healthcare expenditures associated with CDI has demonstrated that direct health care costs and increased length of stay were the main hospital cost drivers. 2 Therefore, preventative measures could reduce national annual hospital-associated costs of CDI, which now approach 7 billion dollars. 8 In addition to direct financial costs, CDI has been shown to negatively impact patient-reported health status in regard to physical, mental and social well-being [2].

In this nationally representative study, we identified a lower incidence of CDI than reported in smaller single institutional cohort studies. Among nearly 5000 patients undergoing cystectomy with urinary diversion, we found that the incidence of CDI was 2.6\%. This rate is lower than prior studies which reported a rate of $8.8 \%$ in a series of 522 patients and $11.7 \%$ in a series of 350 patients and may reflect a more representative national sample $[9,10]$. However, a lower incidence may also relate to methodological differences in capturing CDI which is limited to 30 days after surgery within NSQIP. Underreporting of CDI may also stem from differences in methodology of data abstraction and breadth of available diagnostic tests. In our cohort, stool culture, glutamate dehydrogenase enzyme immunoassay or latex agglutination, PCR assay, stool cytotoxin test, or enzyme immunoassay for toxins A and B were all available modalities; however, they all present with different sensitivities and specificities, which may contribute to institutional variations in reported rates. In studies evaluating the quality of data from NSQIP compared to other national surgical outcomes databases, NSQIP mortality and complication rates were lower than comparable databases, which likely are attributed to how these hospitals systematically monitor 
their surgical outcomes to attenuate complication rates. This discrepancy in outcome may also be attributed to the possibility that nurse abstractors exclude complication rates or mortality rates not directly attributable to a procedure, which is not true of other databases [31]. These differences may also relate to the larger sample size, drawing from over 200 institutions which increases diversity of patient and procedural complexity and reduces variations in local institutions attributed to antibiotic prophylaxis practices, clinical volume, and patient morbidity. A large number of studies regarding CDI rates are nonrandomized and as such, selection bias may contribute to the variability in reported CDI rates [30]. Given these limitations, our study aims to minimize selection bias by including all NSQIP patients undergoing urological surgery for a two-year period from 2015 to 2016 .

Although this is the largest national analysis of postoperative CDI after urologic surgery there are several limitations that require attention. Most notably, through the use of national administrative data, we are unable to account for antibiotic use, as well as type and dosage, both prior to surgery and in the perioperative period. Furthermore, information regarding the outcome of $\mathrm{CDI}$ treatment and a prior history of CDI were unavailable. As CDI is highly contagious within healthcare delivery systems, we are unable to incorporate the local incidence rates at the hospital-level that may further modify postoperative risk. Although the incidence was greatest after radical cystectomy with urinary diversion, we were unable to account for effects that may further alter the host microbiome including the use of neoadjuvant chemotherapy, type of urinary diversion during radical cystectomy, mechanical or antibiotic bowel preparations. Additionally, there are other factors that are not recorded by NSQIP such as the identity of the surgeon, local antibiotic resistance or antibiogram, or hand hygiene that may further modify these outcomes.

\section{Conclusions}

The overall incidence of CDI after urological surgery was low $(0.31 \%)$ but varied substantially by surgery type and was highest after radical cystectomy with urinary diversion $(2.72 \%)$. postoperative CDI was associated with both patient factors, including renal failure and aggregate measures of comorbidity, as well as surgical factors including use of blood transfusion. CDI was associated with significantly longer length of stay.
Author contributions $\mathrm{KN}$ : protocol/project development, data collection or management, data analysis, manuscript writing/editing, DL: protocol/project development, manuscript writing/editing, YB: protocol/project development, manuscript writing/editing, SR: protocol/ project development, manuscript writing/editing, JK: protocol/project development, manuscript writing/editing, PK: protocol/project development, manuscript writing/editing, ML: protocol/project development, data collection or management, manuscript writing/editing.

Funding: None.

\section{Compliance with ethical standards}

Conflicts of interests The authors declared that they have no conflicts of interest.

Ethics approval IRB exemption.

\section{References}

1. Khanna S, Pardi DS (2012) Clostridium difficile infection: new insights into management. Mayo Clin Proc 87:1106-1117

2. Smits WK, Lyras D, Lacy DB, Wilcox MH, Kuijper EJ (2016) Clostridium difficile infection. Nat Rev Dis Primers 2:16020

3. Cunha BA (1998) Nosocomial diarrhea. Crit Care Clin 14:329-338

4. Suljagic V, Miljkovic I, Starcevic S, Stepic N, Kostic Z, Jovanovic $\mathrm{D}$ et al (2017) Risk factors for Clostridium difficile infection in surgical patients hospitalized in a tertiary hospital in Belgrade, Serbia: a case-control study. Antimicrob Resist Infect Control 6:31

5. Yoo SJ (2010) The risk factors of Clostridium difficile colitis in colorectal surgery. J Korean Soc Coloproctol 26:307-308

6. Rodrigues MA, Brady RR, Rodrigues J, Graham C, Gibb AP (2010) Clostridium difficile infection in general surgery patients; identification of high-risk populations. Int J Surg (Lond Engl) 8:368-372

7. Abdelsattar ZM, Krapohl G, Alrahmani L, Banerjee M, Krell RW, Wong SL et al (2015) Postoperative burden of hospital-acquired Clostridium difficile infection. Infect Control Hosp Epidemiol 36:40-46

8. Zhang S, Palazuelos-Munoz S, Balsells EM, Nair H, Chit A, Kyaw MH (2016) Cost of hospital management of Clostridium difficile infection in United States-a meta-analysis and modelling study. BMC Infect Dis 16:447

9. Cotter KJ, Fan Y, Sieger GK, Weight CJ, Konety BR (2017) Prevalence of Clostridium Difficile infection in patients after radical cystectomy and neoadjuvant chemotherapy. Bladd Cancer (Amst Neth) 3:305-310

10. Liu NW, Shatagopam K, Monn MF, Kaimakliotis HZ, Cary C, Boris RS et al (2015) Risk for Clostridium difficile infection after radical cystectomy for bladder cancer: analysis of a contemporary series. Urol Oncol 33(503):e17-22

11. Carignan A, Allard C, Pepin J, Cossette B, Nault V, Valiquette L (2008) Risk of Clostridium difficile infection after perioperative antibacterial prophylaxis before and during an outbreak of infection due to a hypervirulent strain. Clin Infect Dis Off Publ Infect Dis Soc Am 46:1838-1843

12. McDonald LC, Gerding DN, Johnson S, Bakken JS, Carroll KC, Coffin SE et al (2018) Clinical practice guidelines for Clostridium difficile infection in adults and children: 2017 update by the infectious diseases society of America (IDSA) and society for 
healthcare epidemiology of America (SHEA). Clin Infect Dis 66:e1-e48

13. Chiang SR, Lai CC, Ho CH, Chen CM, Chao CM, Wang JJ et al (2018) Prolonged mechanical ventilation assistance interacts synergistically with carbapenem for clostridium difficile infection in critically Ill patients. J Clin Med 7(8):224

14. Rogers MA, Micic D, Blumberg N, Young VB, Aronoff DM (2014) Storage duration of red blood cell transfusion and Clostridium difficile infection: a within person comparison. PLoS ONE 9:e89332

15. Lawley TD, Clare S, Walker AW, Goulding D, Stabler RA, Croucher $\mathrm{N}$ et al (2009) Antibiotic treatment of clostridium difficile carrier mice triggers a supershedder state, spore-mediated transmission, and severe disease in immunocompromised hosts. Infect Immun 77:3661-3669

16. Asempa TE, Nicolau DP (2017) Clostridium difficile infection in the elderly: an update on management. Clin Interv Aging 12:1799-1809

17. Rajashekar A, Perazella MA, Crowley S (2008) Systemic diseases with renal manifestations. Prim Care 35(297-328):vi-vii

18. Koenig SM, Truwit JD (2006) Ventilator-associated pneumonia: diagnosis, treatment, and prevention. Clin Microbiol Rev 19:637-657

19. Kim JL, Park JH, Han SB, Cho IY, Jang KM (2017) Allogeneic blood transfusion is a significant risk factor for surgical-site infection following total hip and knee arthroplasty: a meta-analysis. J Arthroplasty 32:320-325

20. Kaneko K, Kawai K, Tsuno NH, Ishihara S, Yamaguchi H, Sunami E et al (2015) Perioperative allogeneic blood transfusion is associated with surgical site infection after abdominoperineal resectiona space for the implementation of patient blood management strategies. Int Surg 100:797-804

21. Shander A, Javidroozi M, Perelman S, Puzio T, Lobel G (2012) From bloodless surgery to patient blood management. Mt Sinai J Med 79:56-65

22. Tyson MD, Castle EP, Humphreys MR, Andrews PE (2014) Venous thromboembolism after urological surgery. J Urol 192:793-797

23. Wolf JS Jr, Bennett CJ, Dmochowski RR, Hollenbeck BK, Pearle MS, Schaeffer AJ (2008) Best practice policy statement on urologic surgery antimicrobial prophylaxis. J Urol 179:1379-1390
24. Bonkat G, Bartoletti R, Bruyére F, Cai T, Geerlings SE, Köves B et al (2020) EAU guidelines on urological infections 2020. EAU Guidelines. Edn. presented at the EAU Annual Congress Amsterdam the Netherlands 2020. ISBN 978-94-92671-07-3

25. Slimings C, Riley TV (2014) Antibiotics and hospital-acquired Clostridium difficile infection: update of systematic review and meta-analysis. J Antimicrob Chemotherap 69:881-891

26. Miranda D, Mermel LA, Dellinger EP (2020) Perioperative antibiotic prophylaxis: surgeons as antimicrobial stewards. J Am Coll Surg 231(6):766-768. https://doi.org/10.1016/j.jamcollsur g.2020.08.767

27. Tschudin-Sutter S, Braissant O, Erb S, Stranden A, Bonkat G, Frei $\mathrm{R}$ et al (2016) Growth patterns of Clostridium difficile: correlations with strains, binary toxin and disease severity: a prospective cohort study. PLoS ONE 11:e0161711

28. Banaei N, Anikst V, Schroeder LF (2015) Burden of Clostridium difficile infection in the United States. New Engl J Med 372:2368-2369

29. Parker WP, Tollefson MK, Heins CN, Hanson KT, Habermann EB, Zaid HB et al (2016) Characterization of perioperative infection risk among patients undergoing radical cystectomy: results from the national surgical quality improvement program. Urol Oncol 34:532.e13-e19

30. Li X, Wilson M, Nylander W, Smith T, Lynn M, Gunnar W (2016) Analysis of morbidity and mortality outcomes in postoperative Clostridium Difficile infection in the veterans health administration. JAMA Surg 151:314-322

31. Weiss A, Anderson JE, Chang DC (2015) Comparing the national surgical quality improvement program with the nationwide inpatient sample database. JAMA Surg 150:815-816

Publisher's Note Springer Nature remains neutral with regard to jurisdictional claims in published maps and institutional affiliations. 